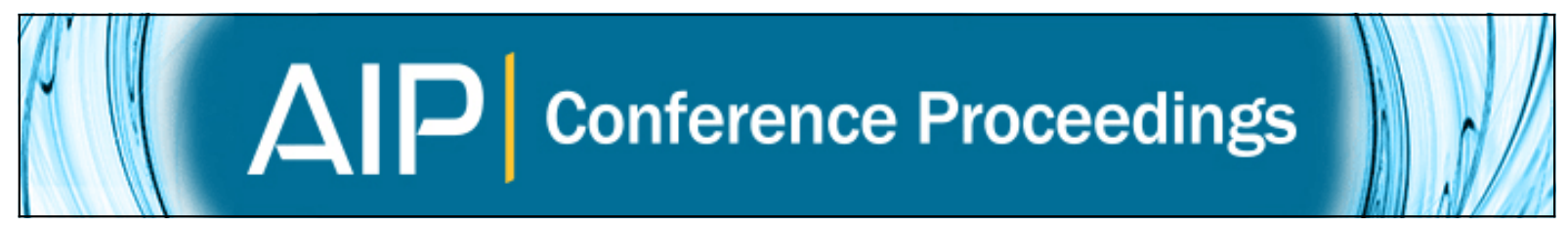

\title{
Colloidal gels under shear: Strain rate effects
}

N. Koumakis, P. Ballesta, R. Besseling, W. C. K. Poon, J. F. Brady, and G. Petekidis

Citation: AIP Conference Proceedings 1518, 365 (2013); doi: 10.1063/1.4794598

View online: http://dx.doi.org/10.1063/1.4794598

View Table of Contents: http://scitation.aip.org/content/aip/proceeding/aipcp/1518?ver=pdfcov

Published by the AIP Publishing

\section{Articles you may be interested in}

Microstructure and nonlinear signatures of yielding in a heterogeneous colloidal gel under large amplitude oscillatory shear

J. Rheol. 58, 1359 (2014); 10.1122/1.4882019

Shear avalanches in metallic glasses under nanoindentation: Deformation units and rate dependent strain burst cut-off

Appl. Phys. Lett. 103, 101907 (2013); 10.1063/1.4820782

Shear thickening and order-disorder effects in concentrated colloids at high shear rates

J. Rheol. 44, 1 (2000); 10.1122/1.551072

Strain rate effects in beryllium under shock compression

AIP Conf. Proc. 78, 427 (1982); 10.1063/1.33296

Effect of Pressure and Strain Rate on the Shear Strength of Beryllium

J. Appl. Phys. 41, 5254 (1970); 10.1063/1.1658658 


\title{
Colloidal gels under shear: Strain rate effects
}

\author{
N. Koumakis ${ }^{1}$, P. Ballesta ${ }^{1}$ R. Besseling ${ }^{2}$, W. C. K. Poon ${ }^{2}$, \\ J. F. Brady ${ }^{3}$ and G. Petekidis ${ }^{1}$ \\ ${ }^{1}$ FORTH-IESL \& Department of Materials Science and Technology, University of Crete, \\ 71110, Heraklion, Crete, Greece \\ ${ }^{2}$ School of Physics and Astronomy, University of Edinburgh, Edinburgh, EH9 3JZ, UK, \\ ${ }^{3}$ Div. of Chem. and Chem. Eng., California Institute of Technology, Pasadena, CA 91125, USA
}

\begin{abstract}
Attractive colloidal particles are trapped in metastable states such as colloidal gels at high attraction strengths and attractive glasses and high volume fractions. Under shear such states flow via a two step yielding process that relates to bond and cluster or cage breaking. We discuss the way the structural properties and related stress response are affected by the shear rate. At low rates colloidal gels yield during start-up shear essentially in a single step, exhibiting a single stress overshoot due to creation of compact flowing clusters. With increasing shear rate a second stress overshoot, linked with further cluster breaking up to individual particles, is becoming more pronounced. We further present the age dependence of the two step yielding and wall slip effects often taking place during rheological experiments of colloidal gels. The latter is related both with the shear rate dependent gel structure as well as the time evolution of the near wall structure.
\end{abstract}

Keywords: Colloidal gels, Colloid-polymer mixtures, Yielding, Wall Slip.

PACS: $82.70 . \mathrm{Dd}, 64.70 . \mathrm{pv}, 83.10 . \mathrm{Mj}, 83.80 . \mathrm{Hj}$

\section{INTRODUCTION}

The addition of non-adsorbing polymer to a colloidal suspension of hard spheres is currently a widely use way to introduce tunable attractions between the colloids ${ }^{5}$ due to depletion phenomenon. The range of attraction depends on the polymer radius of gyration while the strength is proportional to the polymer concentration in the free volume $[1,2,3]$. Colloid-polymer mixtures have a rich phase diagram that depends on the particle volume fraction and polymer concentration, but also on the range of attraction. Increasing interparticle attractions lead to particle bonding and increase bond escape time. creating stable percolated structures that leads to colloidal gelation.

Rheological studies $[4,5,6]$ in colloid-polymer mixtures at high particle volume fractions have shown that an attractive glass exhibits a two step yielding both with increasing strain amplitude in oscillatory shear and increasing strain during a steady step-rate test, as opposed to the single yielding observed in a repulsive glass. The first step was attributed to bond breaking while the second one to cage breaking in the glassy state. As the strain is increased beyond the linear regime, particles are able to change bonding neighbours by escaping from interparticle attraction, thus leading to energy dissipation and partial yielding. When higher strains are applied to the system, the cages are distorted and finally break giving rise to a second yielding point.

While at high volume fractions attractive glasses the two-step yielding reflects bond and cage breaking respectively at lower volume fraction gels cages seize to exist but are replaced by interconnected clusters $[6,7]$. The size of the latter introduces now the second length-scale present in the system, in addition to that of the inter-particle bond range and in analogy with the cage size at volume fractions above the glass transition. The rheology and more specifically yielding response of intermediate volume fraction gels have been studied both under constant attraction strength at different volume fractions and at a constant volume fraction with increasing attraction. Yielding throughout this range, probed both by oscillatory and steady shear, is found to remain a two step process until very low $\varphi$ 's. The first yield strain related with in-cage or intercluster bond braking remains constant for $\varphi>0.3$ while the second yield strain, attributed to braking of cages or clusters into smaller constituents, increases as volume fraction is decreased due to enhancement of structural inhomogeneities in the gel $[6,7]$.

A phenomenon often detected in a wide range of complex nanostructured materials is wall slip which complicates rheological measurements [8,9]. In repulsive colloidal glasses and concentrated suspensions slip has been detected at low shear rates and strongly depends on the particle-wall interactions. 
Since any meaningful rheology requires knowledge of boundary effects and affects the outcome of many industrial processes understanding of its microscopic origins and of rheological consequences is of profound importance.

In the paper we first review some of the pertinent features of yielding response intermediate volume fraction colloidal gels under steady and oscillatory shear and present related phenomena of slip and time dependent structural changes which affect the rheological measurements and mechanical response of such systems. We also present and discuss the shear rate effect on the structure and mechanical response of the gel and the impication this has on rejuvenation protocols. To this end experimental rheological studies have been complemented with Brownian Dynamics (BD) simulations that provide valuable information on the microscopic structure of the gels under shear.

More specifically we study gels at intermediate $\varphi$ $(\sim 0.4)$, which form percolated network of clusters. We study slip and micro-structural evolution at various state points using a combination of rheometry and confocal imaging. The structural and rheological properties of a strong colloidal gel are examined during steady rate shear flow as well as after cessation using rheology and BD simulations.

Through structural analysis of the steady state, with simulations, we find that variation of the applied shear rate produces strong changes in the structure of the gels both during flow and additionally at gel reformation after cessation. Analysis of structural changes is carried out by examination of the empty space or voids in the sample, which can give a quantitative measure of real space heterogeneity. Larger rates are characterized by disperse particles and the total breakage of structures at rest, however, smaller rates show large inhomogeneous structures under flow. The changes in structural properties with shear rate can be attributed to the balance between shear forces and depletion forces, given by their ratio, $\mathrm{Pe}_{\mathrm{dep}}$ [7], which is an estimation of when bonds should break down due to flow. By combination of rheology and simulations, we find that by tuning the way a gel is sheared, one may vary the structure of the resulting gel.

\section{SYSTEMS AND METHODS}

Hard sphere particles are polymethylmethacrylate (PMMA) colloids of various radii $(\mathrm{R}=130-800 \mathrm{~nm}$ from light scattering) stabilized by a thin layer of poly-12-hydroxystearic acid $(\sim 10$ $\mathrm{nm})$. The particles were suspended in different organic solvents: decalin, decalin-tetralin mixture (for refractive index matching), mixture of decalincycloheptylbromide (CHB) (for refractive index and density matching) or octadecene (to minimize evaporation). To avoid shear-induced crystallization the particles have a polydispersity $p \geq 10 \%$. We estimated $\varphi$ by assuming that a spun-down sample was at random close packing, $\varphi_{\text {rcp }}=0.67$.

The gel consists of model hard sphere particle dispersions with the addition of non-adsorbing linear chains resulting in an attraction between particles at contact of $\mathrm{U}(2 \mathrm{R}) / \mathrm{k}_{\mathrm{B}} \mathrm{T} \approx-20$ and an interaction range of $\xi \approx 0.07$ to 0.1 . The linear chains added where polybutadiene for suspensions in tetradecane and octadecene or polystyrene when the solvent was decalin or decalin/tetralin.

The rheometer used was an ARES-HR strain controlled instrument with a force balance transducer 10FRTN1 and $25 \mathrm{~mm}$ cone-plate geometry (0.044 rad). As a standard protocol before each test a high strain dynamic shear rejuvenation $\left(\gamma_{0}=1000 \%, \omega=1 \mathrm{rad} \mathrm{s}^{-1}\right)$ was performed for dynamic tests or high rate steady shear $\left(\&=100 \mathrm{~s}^{-1}\right)$ for steady shear experiments followed by a waiting time of typically 120 s before the beginning of the experiment (unless stated otherwise). Solvent traps were used to minimize the effect of evaporation over our measurement time of a few hours. We also used a controlled stress rheometer (AR2000, TA Instr.) with a cone-plate geometry (radius $20 \mathrm{~mm}$, cone angle $\theta=1^{\circ}$ ) with a modified base on which a glass slide (radius $25 \mathrm{~mm}$, thickness $180 \mu \mathrm{m}$ ) was mounted so that simultaneous confocal microscopy measurements (not shown here) can be performed.

Different surfaces were used with the cone-plate geometries for slip studies, i) In closely indexmatching solvents (cis-decalin, tetralin-decalin and decalin-cycloheptylbromide mixtures) surfaces were roughened by coating and sintering of a disordered monolayer of colloids of radius similar to those in the suspension. ii) For non-index matching solvents (cis/trans-decalin, octadecene, tetradecane) typically used in conventional rheological tests we used the standard rheometer tools (titanium or aluminium) with smooth surfaces where a monolayer of particles was stuck due to van der Waals forces. iii) Finally some experiments were carried out with serrated tools consisting of a home-made cone and plate with roughness of $100 \mu \mathrm{m}$, similar to commercial tools typically used to suppress slip. The home-made tools where tested and calibrated with viscous and viscoelastic standards.

BD simulations were conducted by using a potential-free algorithm for hard sphere interactions [10] with the superposition of a simple AsakuraOsawa potential for depletion attractions with similar parameters. 


\section{RESULTS AND DISCUSSION}

\section{Two Step Yielding in Colloidal Gels}

We first review the two step yielding behavior of intermediate volume fraction gels presented before [7]. The non-linear oscillatory response of the attractive glass and gels at various volume fractions and constant attraction strength $\left(\mathrm{U}(2 \mathrm{R}) / \mathrm{k}_{\mathrm{B}} \mathrm{T} \approx-20\right.$ and $\left.\xi \approx 0.1\right)$ is presented in figure 2 by a dynamic strain sweep (DSS) tests at $\omega=10 \mathrm{rad} \mathrm{s}^{-1}$. Five different volume fractions, spanning the phase diagram from the attractive glass regime at high $\varphi$ down to a very low $\varphi$ gel are seen. At the highest volume fraction, well within the attractive glass region, there are two distinct peaks of $\mathrm{G}^{\prime}$, signifying yielding through the two mechanisms discussed previously [4-7] the first related to bond breaking and the second to cage breaking. As the volume fraction is decreased the second peak attributed to cage breaking, becomes broader and less pronounced, moving to higher strains, until it disappears at the lowest volume fraction.

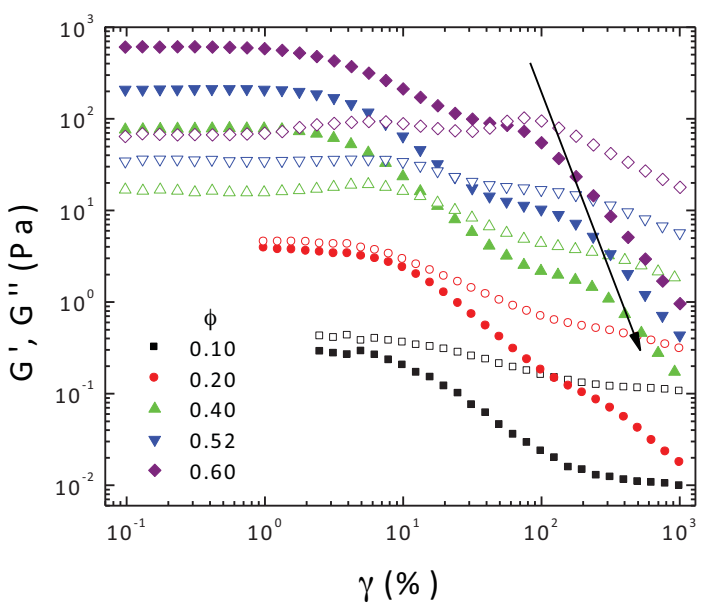

FIGURE 1: Dynamic strain sweeps at $\omega=10 \mathrm{rad} / \mathrm{s}$ for five different volume fractions with equal attraction strength as indicated. The arrow shows the approximate shift of the second G' peak with decreasing volume fraction. Data from [7]

Steady rate (start-up shear) tests were applied monitoring the stress against strain (or equivalently time) [7]. These tests are very similar to the step strain experiments carried out by Pham et al [5] where an instantaneous strain was applied and the resulting stress peak was plotted with changing strain. Step rate experiments are simpler than oscillatory strain sweeps, as the rate remains constant throughout the test and since they are less time consuming could be better in examining the time dependence (ageing) of non-linear properties.
In figure 2 we compare step rate tests for a high $\varphi$ repulsive and attractive glass as well as a lower $\varphi$ gel. In the hard sphere repulsive glass a single stress overshoot is observed. The physical mechanism related with the stress overshoot is related with the storage of elastic energy via a deformation of the structure of the particles or their deformation (in the cage of soft colloids or polymers) which is then release mechanism after the peak leading to shear induced flow. The detailed relation between the stress and the microscopic structure and particle displacements for hard sphere glasses was recently present in [11].

Here we point out that whereas the hard sphere glass at $\varphi=0.60$ shows a single peak, both the attractive gel with $\varphi=0.44$ and the attractive glass at $\varphi=0.60$ reveal two distinct peaks as indicated by the vertical arrows. The single stress overshoot in the case of the hard sphere glass reflects the single yielding mechanism corresponding to cage breaking. In contrast, for the attractive gels and glasses two stress peaks are observed in agreement with the two step yielding processes found in oscillatory measurements.

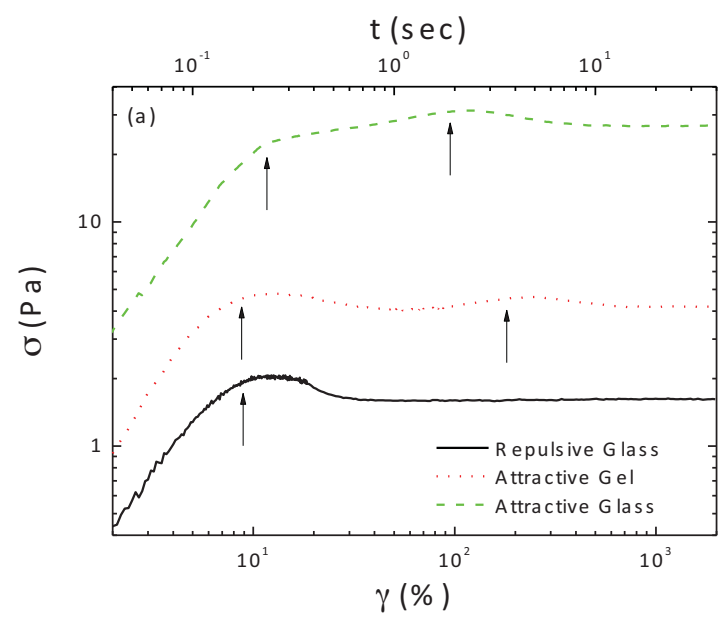

FIGURE 2: Step rate test in a repulsive glass of $\varphi=0.60$, an attractive glass at $\varphi=0.60$ and a gel at $\varphi=0.44$ for a rate of $0.5 \mathrm{~s}^{-1}$. Arrows indicate single and double yielding for repulsive and attractive glass/gel respectively. Data from [7]

As seen by comparing the attractive glass $(\varphi=0.60)$ and the attractive gel $(\varphi=0.44)$, has been shown in detail previously [7], and in agreement with the dynamic measurements (fig. 1) the second peak becomes less pronounced at lower colloid volume fractions and moves to slightly higher strains, until dissipating at the lowest volume fraction measured $(\varphi=0.1)$. 


\section{Shear Rate dependent Structure}

In figure 3, we plot step rate tests with increasing shear rates for an intermediate volume fraction gel, $\varphi=0.44$. The main finding is the evolution of the strength of the second peak which increases with strain rate while the peak value of the second stress overshoot rises much stronger than that of the first one. On the other hand the strain of the second peak is almost shear rate independent. As discussed in more detail elsewhere [7] the stress at the second peak increases with rate following an apparent power law with an exponent of 0.5 , which is almost $\varphi$ independent. At the same time the second yield strain remains essentially constant for gels at intermediate volume fractions $(\varphi=0.3-0.58)$.

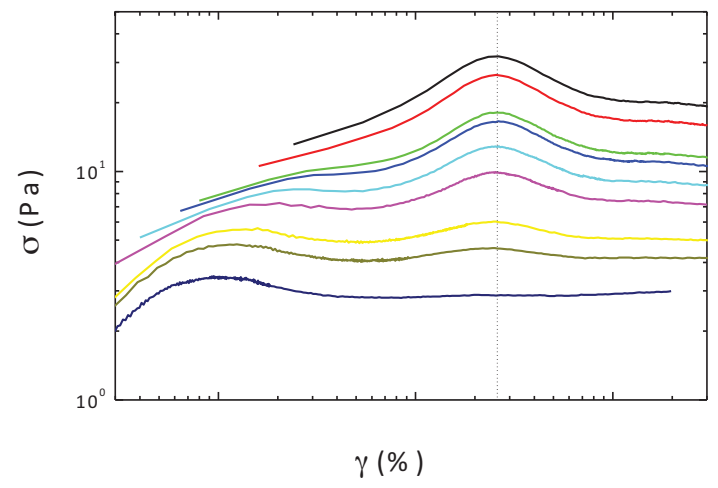

Figure 3: Step rate tests with increasing rates $(0.1,0.5 .1,3,5,8,10$, $\left.20,30 \mathrm{~s}^{-1}\right)$ from bottom to top for $\varphi=0.44$ and the same rates $(0.1-10$ $\left.\mathrm{s}^{-1}\right)$ (data from ref [7])

A physical mechanism that can explain such shear rate dependence is the following as discussed in [7]. At the lowest rates the time scale for the creation of bonds is much shorter than the inverse shear rate. Hence after the first yield point, there is time for rotation and restructuring of clusters with minimum internal bond breaking. Therefore at the lowest rates, the first yield mechanism dominates over the second one which at the limit of zero shear rates is essentially absent (fig. 3). At this limit inter-cluster bonds may break temporarily under shear so that broken clusters can flow past each other while under shear bonds may reform and re-brake.

As higher rates the imposed shear is able to break an increasing amount of clusters which then under flow start colliding with each since there is no time available for restructuring. This causes a kind of jamming of clusters and an eventual stress relaxation and energy dissipation via further breakage of clusters to smaller pieces. This mechanism causes the appearance of the second peak and its enhancement with increasing shear rate.
The first yield strain in attractive glasses and gels has been rationalized using the Kramer's type escape arguments [6], where the probability of a particle to escape from attractive well increases with shear induced displacements. Along this line a simple argument has been suggested [7] to describe the shear rate dependence of cluster breaking into individual particles by introducing a dimensionless number, $\mathrm{Pe}_{\mathrm{dep}}$. This suggests that a cluster would break for rates above which the drag force acting on a particle becomes larger than the depletion attraction. Hence in a two particle cluster when one of them is displaced by shear flow it may drag along the other one if the viscous drag $\mathrm{F}_{\text {visc }}$ is smaller than the bonding force, $\mathrm{F}_{\text {dep }}$. If however we have $F_{v i s c}=6 \pi \eta R v>F_{d e p}$ the bond will break. The relative velocity in a shear flow for two particles in contact is of the order of $\& R$. This argument leads to the introduction of a dimensionless number (called $P e_{\text {dep }}$ ) using a simple ramp potential we have [7]:

$$
F_{v i s c} / F_{d e p}=\frac{12 \pi \eta \xi R^{3}}{U_{d e p(r=2 R)}} \& \equiv P e_{d e p}
$$

This number indicates the rates above which cluster are expected to fully break. Since diffusion and hydrodynamic lubrication effects were ignored and this is essentially applicable for strong attractions.

Moreover the ratio $P e / P e_{d e p}=\frac{U_{d e p(r=2 R)}}{k_{\mathrm{B}} \mathrm{T}}(2 \xi)^{-1}$ relates shear rate needed to fully break clusters to the conventional Peclet number, $P e=\not \mathbb{F}_{B}=\not R^{2} / D_{0}$. Hence a $\mathrm{Pe}_{\mathrm{dep}}=1$ where clusters are expected to fully break corresponds in the present experimental system to $\mathrm{Pe} \approx 117$. Such shear rates are however beyond the values reached in the present work, where a $\mathrm{Pe}=1$ corresponds to $\&=30 \mathrm{~s}^{-1}$ suggesting that clusters may not be fully broken here.

To investigate further the above picture and gather microscopic structural information for the colloidal under we have performed Brownian Dynamics simulations as well as confocal microscopy under shear. While a complete study will be presented elsewhere [12] here we present some data from BD simulations. Figure 4 shows an evolution of the gel structure with increasing shear rate once the steady state has been reached. In agreement with the aforementioned confocal microscopy experiments, and the arguments presented above we see that as the shear rate is increased the steady state structure consists of progressively smaller clusters. We note also that at the limit of small shear rates a densification of the clusters is observed, manifested also by an increase of the free void volume. This structural change corresponds to the shear rate regime where no second stress overshoot is 
detected (fig. 3) suggesting that such rheological response is due to the free flowing densified clusters. Once the shear rate increases, as discussed above, clusters collide with each other and break to smaller pieces $(\mathrm{Pe}=10$ in fig. 4) and only at very high $\mathrm{Pe}$ clusters completely break to individual particles. Such structural changes with shear rate can be quantitatively rationalized by the $\mathrm{Pe}_{\text {dep }}$ defined above as will be shown in a future publication [12].
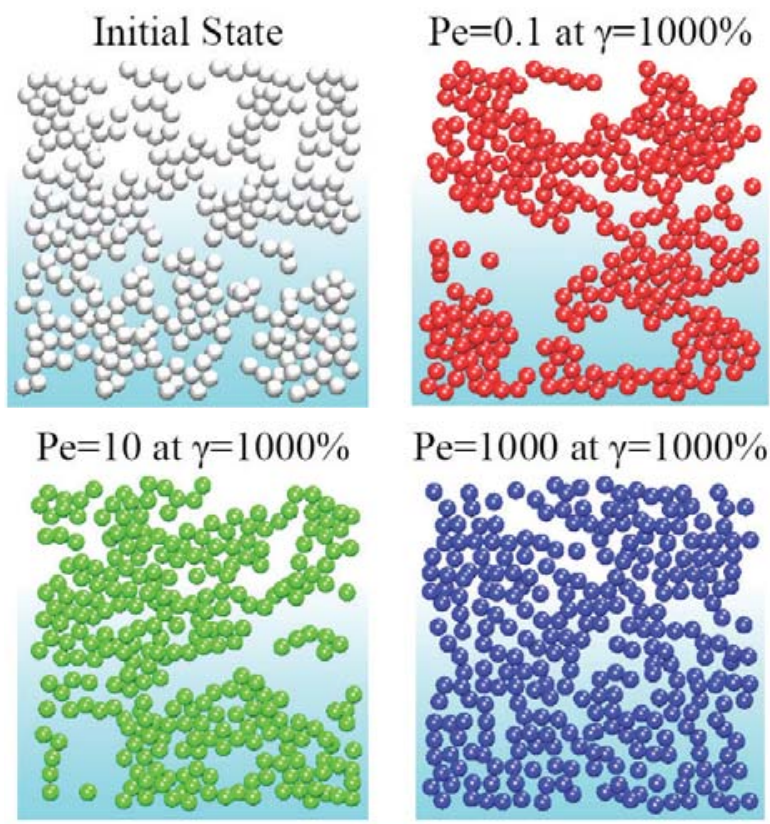

Figure 4: $\mathrm{BD}$ simulation images showing one diameter thick slices of long time configurations with $\varphi=0.44, \operatorname{Udep}(2 R)=-17 \mathrm{k}_{\mathrm{B}} \mathrm{T}$ and $\xi=0.1$ at rest and under shear as labelled.

We further briefly review the consequences of ageing of the gel on the linear viscoelastic properties and the two step yielding response [7]

In figure 5 we show the evolution of G' and G', at $\omega=10 \mathrm{rad} \mathrm{s}^{-1}$ for a gel with $\varphi=0.44$. The elastic modulus clearly increases with waiting time, $t_{w}$, following a weak power law while the viscous modulus slightly decreases. We also measured the non-linear response during step-rate experiments at a rate of $0.5 \mathrm{~s}^{-1}$ for different waiting times after rejuvenation. Although the peak stresses increase with waiting time, the second one shows a more pronounced rise. Since the first peak corresponds to the breaking of bonds its height represents the stress that can be stored in the network before it is released via inter-cluster bond breaking. The subsequent decay of the stress is therefore related to the number of bonds that break. The height of the first peak, $\sigma_{1}$, follows the increasing trend of the elastic modulus with waiting time. The value of $\sigma_{1}$ corresponds approximately to the stress the network can withstand before it starts being disrupted. Its increase with time suggests that either the stress of bond breaking or the number of bonds in the network increase. The stress of the individual bond is unlikely to change in such long times, as bond formation time is of the order of $10^{-2} \mathrm{~s}$ as previously calculated from $\mathrm{Pe}_{\mathrm{dep}}$. Hence the increase of the elasticity and $\sigma_{1}$ should be attributed to the coarsening of the gel and for the case of $\sigma_{1}$, the higher number of inter-cluster bonds. The strong correlation of $\sigma_{1}$ with the aging behaviour of $\mathrm{G}^{\prime}$ is perhaps to be expected, as the initial increase of stress at strains smaller than the first yield point in a step rate test is determined by the linear moduli of the related system at rest.

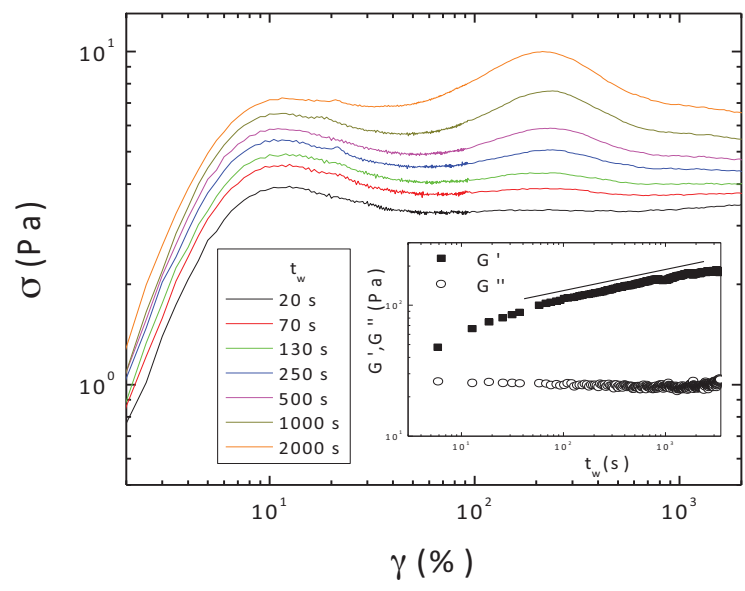

Figure 5: Step rate tests showing stress response versus strain at a rate of $0.5 \mathrm{~s}^{-1}$ for the $\varphi=0.44$ gel for different delay times $(20,70$, $130,250,500,1000,2000 \mathrm{~s}$ from bottom to top). Inset: Linear viscoelastic measurements at $\omega=10 \mathrm{rad} \mathrm{s}^{-1}$ for the $\varphi=0.44 \mathrm{gel}$. The solid line is a power law of 0.13 . (data from ref. [7])

The second peak represents the breaking of clusters/cages. The fact that the second peak becomes larger than the first with time indicates that the strengthening of the gel occurs not only by increasing the number of particle bonds, but more importantly due to restructuring and creation of denser and stronger particle clusters. As discussed, since bond formation time at these attraction strengths should be very small, this rather long-time scale suggests large scale, or long time structural changes. In other words, after the initial quench to the gel state the sample slowly progresses to deeper energetic minima via single particle, as well as, cooperative motions.

\section{Slip behavior and time dependence}

We finally present the slip behavior in colloidpolymer gels. While the data shown above are taken under conditions of no-slip, very often attractive gels 
exhibit significant wall slip that can not be avoided unless very rough serrated plates are used.

Below we normalized the shear stress and moduli by $\bar{\sigma}=\sigma R^{3} / k_{B} T, \quad \bar{G}^{\prime}\left(\right.$ or $\left.\bar{G}^{\prime \prime}\right)=G^{\prime}\left(\right.$ or $\left.G^{\prime \prime}\right) R^{3} / k_{B} T$ respectively, in order to allow comparison of rheological data from samples with different $\mathrm{R}$ and $\mathrm{c}_{\mathrm{p}}$. The normalized shear rate is given by the Péclet number as above, using however in the diffusion coefficient $\mathrm{D}_{0}$ the viscosity of the polymer solution at concentration $c_{p}$ instead of the solvent one.

Figure 6 shows flow curves (up and down sweeps) measured in a cone-plate geometry with smooth or serrated bottom plate (roughness of about $100 \mu \mathrm{m}$ ) for the same sample in tetradecane $\left(\varphi=0.45, c_{p}=0.5 c^{*}\right)$. The flow curves measured with serrated surfaces have a Herchel-Bulkley (HB) form, $\sigma=\sigma_{y}+\alpha$ \& $^{2}$, with $\sigma_{\mathrm{y}}$ the bulk yield stress and $\mathrm{n}$ an exponent typically ranging between 0.4 and 0.7 . However, with smooth surfaces, the stress drops at low \& below the HB behavior indicating wall slip as in previous studies. The low shear rate regime of the flow curve can then be fitted by a Bingham type equation, $\sigma=\sigma_{s}+\eta \ell \&$ where $\sigma_{\mathrm{s}}$ is the slip stress and $\eta$ an apparent viscosity.

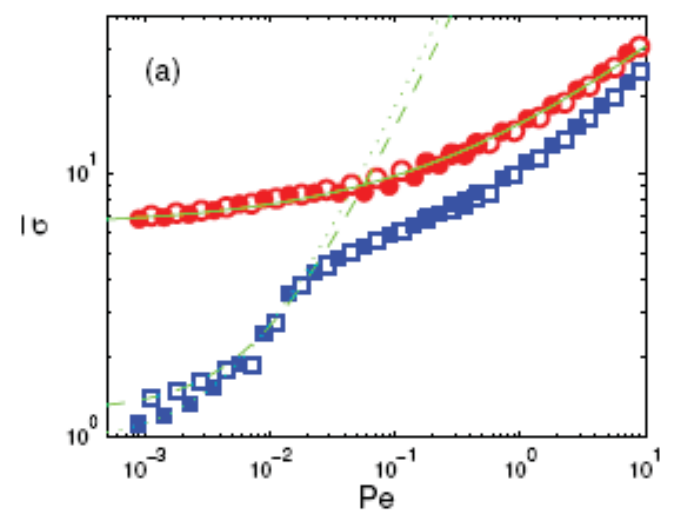

Fig. 6: Flow curves for colloid-polymer gels with $\varphi=0.45$, and $\mathrm{c}_{\mathrm{p}}=0.5 \mathrm{c}^{*}$ (contact potential $\mathrm{U}_{0}=-24 \mathrm{k}_{\mathrm{B}} \mathrm{T}$ ) in tetradecane with smooth tools, where a monolayer of particles are adsorbed due to van der Waals interaction. Flow curves, $\sigma$ versus Pe, with smooth (squares) and serrated (circles) plates, going from high to low shear rates (open) and from low to high (filled).

Contrary to hard spheres glasses [9], in colloidal gels a hysteresis has been detected especially at low rates [13]. Hence here we present data both flow curve data both sweeping down and sweeping up the shear rate. As seen in fig. 6 when slip is present at low rates stresses are larger for the flow curves where the rate is decreased compared to those where the rate is increased. This hysteresis is due to the time-dependent rheology (thixotropy) of the system which we have further studied with small-amplitude oscillatory shear.
Soon after (typically in less than 10s) rejuvenation, during which the gel was shear melted $\left(G^{\prime}<G^{\prime \prime}\right)$, solid like behavior was recovered $\left(\mathrm{G}^{\prime}>\mathrm{G}^{\prime \prime}\right)$, followed by a weak increase of $\mathrm{G}^{\prime}$ and $\mathrm{G}^{\prime \prime}$ typical for attractive systems due to network restructuring. However, after some time (depending on the volume fraction and attraction strength) the increase is followed by a decrease of both $\mathrm{G}^{\prime}$ and $\mathrm{G}^{\prime \prime}$ by more than an order of magnitude towards a state with similar $G^{\prime}, G^{\prime \prime}$ values. This decrease of the elastic modulus with time is clearly different from the aging behavior shown above for systems that exhibit no slip (fig. 5). Note, that for the samples that show the no-slip flow curves (fig 6) where serrated surfaces of roughness much larger compared to the particle size were used, the normal time dependence of fig. 5 is recovered with $\mathrm{G}^{\prime}$ increasing and $\mathrm{G}^{\prime \prime}$ decreasing.
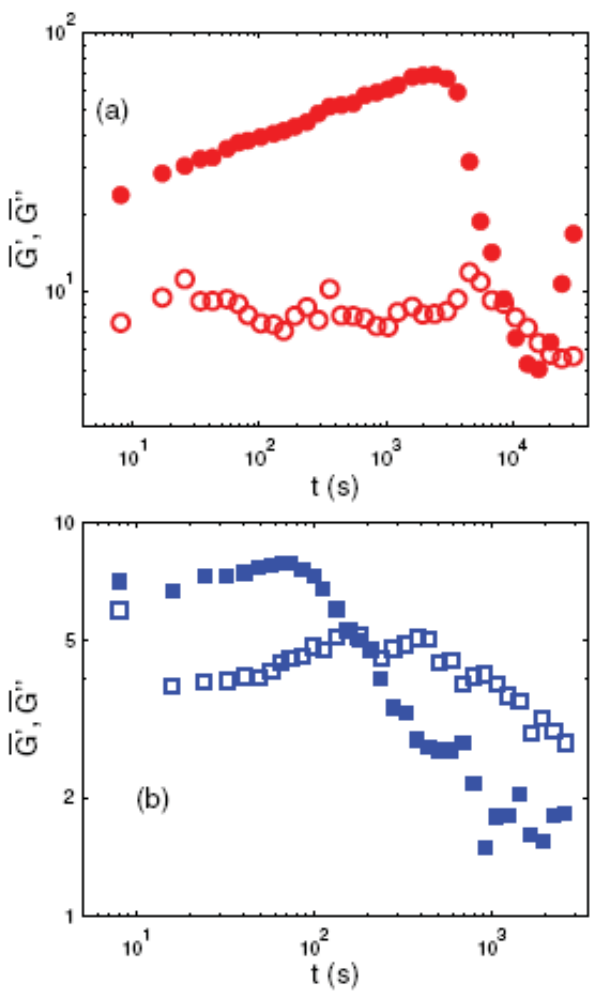

Fig. 7: (a) Dynamic time sweeps at $\mathrm{f}=1 \mathrm{~Hz}$ with $\gamma_{0}=0.1 \%$ : $\mathrm{G}^{\prime}$ and $\mathrm{G}^{\prime \prime}$ versus time (filled and open symbols respectively) for samples with $\mathrm{p}=24 \%$ and $\mathrm{U}_{0}=-18 \mathrm{k}_{\mathrm{B}} \mathrm{T}$ (a) $\varphi=0.35$, (b) $\varphi=0.25$.

The time dependent behavior leading to an apparent drop of G' below that of G' ' is also affected by the particle volume fraction suggesting that it might be driven by a transient network collapse. Fig. 7 shows the time dependence of $\mathrm{G}^{\prime}$ and $\mathrm{G}^{\prime \prime}$ for two samples with concentrations lower than the $\varphi=0.45$ sample already discussed, but with similar $\mathrm{U}_{0}$. These samples, 
at $\varphi=0.35$ and 0.25 , show clear evidence of slip with $\mathrm{G}^{\prime}$ becoming lower than $\mathrm{G}^{\prime \prime}$ after a certain characteristic time which is smaller for smaller volume fractions.

Such time-dependence of viscoelastic properties is reminiscent of observations in sedimenting suspensions of attractive colloids [15] where slip is suddenly detected at the cell walls due to the gel restructuring that lead to a decrease of the number of gel network - wall contact points. The shear rate

\section{CONCLUSIONS}

We have presented the main findings of two step yielding in colloidal gels and specifically discussed the shear rate dependence of the stress during start up shear and the underlying mechanisms due to structural changes under shear.

At the limit of low shear rates the second overshoot attributed to cluster braking is absent due to the altering of their size and structure during flow beyond the first stress overshoot that brakes intercluster bonding. More specifically at the low shear rate regime the clusters under flow densify while larger empty voids are created as BD simulations have revealed. This structural change enables free flow of clusters without a further break up and thus eliminating the second stress overshoot.

At intermediate rates the second overshoot appears due to jamming of the clusters and their subsequent breakup to smaller pieces. Such behavior is becoming more pronounced with increasing shear rate as Brownian activated cluster restructuring is less probable. Eventually at large rates the clusters may totally shear melt to individual particles. A dimensionless number, $\mathrm{Pe}_{\mathrm{dep}}$ indicating where viscous drag forces become equal to depletion attraction provides an estimate of the shear rate beyond which the gel may be fully decomposed to individual particles.

We have also presented the ageing behavior of the colloidal gels and the way the second stress overshoot increases in strength with gel age due to a coursing of the gel structure.

We have finally discussed wall slip phenomena that are detected in such colloidal gels when surface roughness and/or particle-wall attraction is not strong enough. Under intermediate particle-wall attractions or surface roughness a delayed slip is observed due to the time evolution of the near wall gel structure. In this case slip is caused by the reduction of the number of bonds between bulk and surface as the cluster size dependent gel structure and its time evolution are thus expected to determine also the wall slip behavior detected at low shear rates. Such study complemented with rheo-confocal experiments that provide the flow profile as well as the gel microstructure as a function of shear rate will be the subject of a separate work [14].

increases. Therefore, one must use tools with roughness orders of magnitude larger than the particle size to effectively prevent slip. This phenomenon should be expected in every aggregating system such as attractive colloidal suspensions and clearly highlights, in agreement with previous studies that special care should be taken when interpreting rheological data from complex multi-component fluids.

\section{ACKNOWLEDGMENTS}

We thank A. B. Schofield for particle synthesis. We acknowledge EU funding through ToK 'Cosines' (MTCD-CT-2005-029944) and FP7 'Nanodirect' (NMP4-SL-2008-213948).

\section{REFERENCES}

[1] S. Asakura and F. Oosawa, The Journal of Chemical Physics, 1954, 22, 1255-1256.

[2] D. G. A. L. Aarts, R. Tuinier and H. N. W. Lekkerkerker, Journal of Physics Condensed Matter, 2002, 14, 7551-7561.

[3] W. C. K. Poon, Journal of Physics Condensed Matter, 2002, 14, R859-R880.

[4] K. N. Pham, G. Petekidis, D. Vlassopoulos, S. U. Egelhaaf, P. N. Pusey and W. C. K. Poon, Europhysics Letters, 2006, 75, 624630

[5] .K. N. Pham, G. Petekidis, D. Vlassopoulos, S. U. Egelhaaf, W. C. K. Poon and P. N. Pusey, Journal of Rheology, 2008, 52, 649-676.

[6] M. Laurati, S.U. Egelhaaf and G. Petekidis, J. Rheology, 55, 673 (2011)

[7] N. Koumakis and G. Petekidis Soft Matter, 7, 2456, (2011)

[8] P. Ballesta, R. Besseling, L. Isa, G. Petekidis and W.C.K Poon Phys. Rev. Lett. 101, 258301 (2008)

[9] P. Ballesta, G. Petekidis, L. Isa, W. C. K. Poon and R. Besseling J. Rheology 56(5), 1005-1037 (2012)

[10] R. Foss and J. F. Brady, J. Rheol. 44, 629, (2000)

[11] N. Koumakis, M. Laurati, S.U. Egelhaaf, J. F. Brady and G. Petekidis Phys Rev. Lett. 108, 098303 (2012)

[12] N. Koumakis et al, in preparation (2012)

[13] P. Ballesta et al. in preparation (2012)

[14] R. Buscall et al., J. Rheol., 1993, 37, 621--641.

[15] J.-M. Condre, C. Ligoure, and L. Cipelletti, J. Stat. Mech., 2007, P02010. 